This document is the accepted manuscript version of the following article:

Angst, M., \& Hirschi, C. (2017). Network dynamics in natural resource governance: a case study of Swiss landscape management. Policy Studies Journal, 45(2), 315-336. https://doi.org/10.1111/psj.12145

\title{
Network Dynamics in Natural Resource Governance: A Case Study of Swiss Landscape Management
}

Policy Studies Journal 


\begin{abstract}
Structural characteristics of social networks have been recognized as important factors of effective natural resource governance. However, network analyses of natural resource governance most often remain static, even though governance is an inherently dynamic process. In this article, we investigate the evolution of a social network of organizational actors involved in the governance of natural resources in a regional nature park project in Switzerland. We ask how the maturation of a governance network affects bonding social capital and centralization in the network. Applying separable temporal exponential random graph modeling (STERGM), we test two hypotheses based on the risk hypothesis by Berardo and Scholz (2010) in a longitudinal setting. Results show that network dynamics clearly follow the expected trend toward generating bonding social capital but do not imply a shift towards less hierarchical and more decentralized structures over time. We investigate how these structural processes may contribute to network effectiveness over time.
\end{abstract}

KEYWORDS: natural resource governance, policy networks, social network analysis 


\section{Introduction}

Social networks play an important role in natural resource governance. This has been recognized by a number of studies that have investigated how the structure of social networks affects the effectiveness of natural resource governance (eg., Bodin and Crona 2009; Carlsson and Sandström 2008; Janssen et al. 2006; Newig, Günther, and Pahl-Wostl 2010), for example, by discussing the role of social capital in facilitating cooperation or differences between more or less hierarchically organized networks.

Still, one important aspect has not been sufficiently addressed in this context. As every form of social networks, governance networks are "dynamic by nature" (Snijders, van de Bunt, and Steglich 2010, 44). They evolve and change over time while they perform different functions and face equally dynamic governance challenges (Carlsson and Sandström 2008; Bodin and Crona 2009). It is therefore not only crucial to find out what network structures are associated with effective governance, but also how and why these network structures change over time.

In a variety of regional contexts, both scholars and policymakers have endorsed and advocated a more local and bottom-up approach to natural resource governance, especially over the last 25 years. These resulting policies have led to the emergence of regionally specific policy networks comprised of public and private actors managing local resources ranging from watershed estuaries (Berardo and Scholz 2010) to urban parks (Ernstson, Sörlin, and Elmqvist 2008), landscape (Hirschi 2010) and forest biodiversity (Borg, Toikka, and Primmer 2015) in a rather decentralized way.

Yet these networks are not static but evolve over time. They start out as nascent networks, trying to establish management objectives or visions for development. By doing so, they have to gather information and coordinate the views of a sometimes very diverse set of actors. As they mature over time and attempt to put their visions into practice, they are bound to face new problems and challenges. Addressing these problems and challenges requires enhanced cooperation between 
actors.

To further enhance our understanding of these new governance approaches and in particular the relationship between the effectiveness of governance and the underlying network structure recognized in the literature, it is crucial to investigate the maturation of governance networks and associated changes in structural characteristics. Notably bonding social capital and centralization are two commonly named structural characteristics associated with network effectiveness. We focus on this dynamic aspect of networks in natural resource governance and aim to answer the following research questions:

How do social networks in natural resource governance change over time? How do changes from nascent to mature network stages of network development affect the structure of social capital and centralization in the network?

Up to now, the research stream on social networks and natural resource governance has remained strongly case-study-oriented and, if comparative, largely cross-sectional. It has mainly been providing single snapshots of individual networks at one specific point in time (Bodin and Prell 2011). Berardo and Scholz (2010) provide a notable exception but overall, there has been little research until now into how empirically observable networks evolve over longer periods of time and what drives their structural development, even though there has been a clear recognition that longitudinal studies of this kind are needed (Bodin and Crona 2009; Lubell 2015). In this study, we make two contributions in this regard. Methodologically, we show that recently developed methodological advances are well suited to analyze policy network change over time. Theoretically and empirically, we aim to advance the knowledge about central mechanisms associated with policy network dynamics and change over time, focusing on bonding social capital and centralization.

Why is it especially important to gain an understanding of network dynamics in natural resource governance? We see three main reasons:

First, from a governance perspective, the importance of a dynamic perspective cannot be un- 
derestimated. Governance, understood as new forms of political steering beyond traditional hierarchies and command-and-control instruments (Rhodes 1997), calls for flexibility in policymaking necessary to ensure and enhance capacities such as the adaptability of policy solutions to changing context conditions (Adger 2003), the inclusion of the relevant stakeholders (Meadowcroft 2004) and the updating and incorporation of knowledge into the policy process (Sanderson 2002). These characteristics highlight the importance of understanding governance as a dynamic process that allows for a constant adjustment, deliberation and learning.

Secondly, network dynamics are of pivotal importance in the governance of natural resources. Especially the governance of complex social-ecological systems (SES) faces profound uncertainties about the influence of human action on biophysical processes (Anderies, Janssen, and Ostrom 2004) and highly complex system dynamics (Underdal 2010). This has led scholars to emphasize the importance of adaptability (Folke et al. 2005), learning (Armitage, Marschke, and Plummer 2008), transformability and resilience (Walker et al. 2004) in the governance of SES over longer time periods. An understanding of how network dynamics play out and affect network effectiveness under changing circumstances is therefore crucial (Lubell 2015, 43).

Thirdly, a dynamic perspective offers also analytical advantages over a static or cross-sectional research design. Information about time-specific variables allows to go beyond correlations between the coexistence of the relevant variables at one point in time and to explore causal mechanisms based on observable changes in these variables over time (Gerring 2010).

In our attempt to advance our understanding of network dynamics in natural resource governance, we draw upon recent theoretical and methodological developments and innovations:

Lubell (2013) provides a theoretical foundation for conceptualizing governance network development with the Ecology of Games (EG) framework. Situated in the wider Institutional Analysis and Development (IAD) framework (Ostrom 2011), the EG framework understands the unique setting of actors, issues and institutions which emerges within a policy arena as a complex adaptive system which evolves over time (Lubell, Robins, and Wang 2014). Within this framework, a 
very useful hypothesis for understanding network development has been put forward by Berardo and Scholz (2010) with their risk hypothesis, which connects the structural changes during the maturation of a network to a change in the underlying local ecology of games.

Methodological innovations in the wider field of network science provide increasingly sophisticated statistical models of networks, which is especially valuable for empirical research in the EG framework (Lubell 2013, 553). Particularly, methodological developments have continued to push the boundaries of longitudinal network modeling. This enables us to utilize a separable temporal exponential random graph model (STERGM) in our dynamic analysis of natural resource governance, a recently developed specification of exponential random graph models (ERGMs) (Krivitsky and Handcock 2014), allowing for statistical tests of dynamic network hypotheses.

Despite the (rather recent) availability of theories and methodological tools to investigate hypotheses on network dynamics, there is still a wide lack of empirical studies that fully use the theoretical and methodological potential of these innovations to advance our understanding of governance network dynamics. Our study provides such an empirical analysis of how a social network in natural resource governance evolves when the network matures.

Based on the risk hypothesis by Berardo and Scholz (2010) we test for the presence of two important structural drivers of network development in maturing social networks for natural resource governance, namely the opposing trends toward decreasing centralization and increasing closure.

The new Swiss regional nature park (RNP) policy offers a well suited empirical test case for our analysis. RNPs are a relatively new but essential element of the Swiss strategy for regional development and sustainable landscape management. As a policy program, the promotion of RNPs has been enshrined in a revision of the Federal Act on the Protection of Nature and Cultural Heritage in 2007. Regional nature parks can be seen as specific projects set up to manage landscape as a resource in a more sustainable way (Gerber and Knoepfel 2008).

Empirically, we will investigate and analyze the social network established with the RNP project in the region of Thal in the Swiss canton of Solothurn. The involvement and participation 
of all actors in a region is an important part of the RNP policy and prescribed for the establishment as well as the management of a RNP. The establishment of a park, thus, combines top-down guidance by higher administrative levels with required bottom-up local initiatives (Hirschi 2010). This way, the establishment of a governance network surrounding a RNP consisting of a wide variety of actors is not only a goal, but also a distinctive characteristic of RNPs.

In the text that follows, we first introduce the fundamental concepts of network maturation in natural resource governance using the EG framework. We then derive two hypotheses about the expected role of two structural drivers of network development and set them apart from actorattribute-based effects. Next, we will present the empirical case of the RNP of Thal, introduce our methods statistical analysis based on STERGM) and discuss our results. Finally, we will discuss our findings in the light of the recent literature on network dynamics and natural resource governance.

\section{Evolving social networks in natural resource governance}

A profound argument for incorporating networks into the analysis of political phenomena is that individual action is embedded in a rich system of social relations. Even phenomena such as markets which have become almost synonymous with rational choice analysis steeped in methodological individualism have been shown to display such embeddedness (Granovetter 2005). Policy-making for natural resource governance is therefore understood to happen within a complex system of interdependent interactions (Lubell et al. 2012).

A theoretical approach well-suited for the analysis of such complex systems of interdependent interactions is the Ecology of Games (EG) framework (Lubell 2013). The EG framework focuses on political actors involved in complex governance arrangements to resolve collective-action problems by engaging in bargaining. This setting can be represented by multiple, simultaneous policy games in a geographically defined area (Lubell, Robins, and Wang 2014). Embracing complexity, 
the EG approach incorporates a number of theories from social and systems sciences and lends itself particularly well to an analysis of network structures. Furthermore, it allows to formulate hypotheses on assumed dynamics in such network structures because actors within a local EG are understood to participate in institutions that are subject to constant change. The survival of institutions is based on how well they prove to solve collective action problems and are perceived as fair (Lubell 2013).

What happens to the structure of a governance network during its evolution? We suggest that answers can be found by looking at different stages of network development and what they imply for the local ecology of games. Thus, we broadly conceptualize two stages in the development of a governance network, differentiating the network's creation as a nascent network from its subsequent maturation. Between these two stages, a shift in the predominant collective action problem from coordination to cooperation occurs.

In nascent networks, most of the activity is related to the development of network structures and less focus is laid on network performance toward a common goal (Kenis and Provan 2009). We therefore expect actors in a nascent network to mainly be concerned with coordination problems. These occur when actors are seeking innovative solutions for goals they can roughly agree upon. The focus lies on gathering information and agreeing on a common strategy for dealing with a problem (Berardo 2014, 237).

As the network matures, it is expected to shift its focus onto goal achievement. This brings about a shift towards more complex cooperation becoming the more important type of problem faced by actors. The role of social networks in facilitating cooperation is in many ways a recurring theme in the literature on social networks and their role in natural resource governance (eg. Robins, Lewis, and Wang 2012). In comparison to coordination problems, uncooperative behavior is much more likely in cooperation problems. A classic example for this in natural resource management is the overuse of common-pool resources (Berardo 2014, 237).

During this shift from coordination to cooperation, what are the fundamental mechanisms of 
change at play in a network? Broadly, two main groups of mechanisms can be identified.

On the one hand, non-structural or dyadic-independent mechanisms are related to individual agency often based on actor attributes. In fact, many network dynamics may in fact be largely based on actor attributes. If the focus lies on studying these types of mechanisms, processes of social influence and social selection are commonly differentiated (Robins, Lewis, and Wang 2012). One example of a social selection mechanism at work in a network would be a high tendency for actors who have similar views on an issue (i.e., share an attribute) to become friends (i.e., form a tie).

On the other hand, structural or dyadic-dependent effects refer to the fact that ties in networks may change solely because of the configuration of other ties. Consider for example a network where there is a strong dynamic of triangular closure (Snijders et al. 2006, 101). This means that two actors who have a cooperation partner they share (i.e., share a tie) are more likely to cooperate also with each other (i.e., form a tie) than two random, unrelated actors. Thus, there are sources of change in the network that solely depend on the distribution of ties.

This distinction between non-structural and structural effects is crucial, both methodologically as well as theoretically. In the following, we test two hypotheses that are based on the general risk hypothesis by Berardo and Scholz (2010). The risk hypothesis predicts that network structure depends on the relative presence of coordination versus cooperation problems. Its predictions relate mostly to structural effects as will be shown in the next section. Therefore, in order to empirically test hypotheses derived from it, we have to separate non-structural from structural effects. As will be shown further below, this also influences our choice of methods.

\section{The role of risk in network development}

With their risk hypothesis, Berardo and Scholz (2010) introduce a very useful argument about how actors choose partners in a network depending on the structure of risks in the local ecology of games. Risk in this context relates to a decision actors have to make when forming new ties 
in a policy network. Building and maintaining ties to other actors in a policy network is costly. However, relying on only a small number of non-redundant contacts can be risky for an actor as well (non-redundant contacts provide an actor with access to parts of a network that can not be reached in the same way otherwise). The risk hypothesis argues that there is a difference in the level of this risk depending on the nature of the collective action problems in a network and that this shapes the choices of actors to build or maintain ties. The risk for individual actors (and, thus, the overall level of risk in the network) is especially high in situations where the problems in a network are complex and have far-reaching consequences. This creates a situation where there is a high incentive for a given actor to lie or cheat. Cooperation in a network thus becomes dependent on high credibility of information. From the perspective of an individual actor, relying on multiple sources of information can augment the credibility of information. Actors are therefore expected to change their strategy. Maintaining more redundant ties and building stronger relationships helps to encourage a climate where cheaters can be punished and credible commitments make cooperation easier. Therefore, in situations of high-risk cooperation dilemmas the importance of bonding social capital increases.

On the other hand, networks where low-risk coordination dilemmas prevail are expected to develop bridging structures with so-called centralized information hubs. If other actors can be expected to cooperate because problems are simple to solve, there is not much to be gained by non-cooperation and the focus lies on cooperation and accessing new information. The costs of maintaining redundant contacts outweigh the potential risks in this scenario. Actors are thus expected to build efficient ways of information exchange in a network by relying on few central actors (shortening path lengths) and bridging social capital. Bridging social capital increases the chance of an actor to gain access to new sources of information and innovation.

The risk hypothesis is not only theoretically well grounded in the EG framework and gives detailed specifications to the sometimes vague concepts of bonding and bridging social capital but also applies to a dynamic perspective on network development. Even though Berardo and Scholz 
(2010) have tested their hypothesis empirically only on newly formed networks characterized by low-risk dilemmas in the case of the management of American estuaries, they are explicitly concerned with possible long-term developments, hinting at possible consequences of their hypothesis for more mature networks.

Further empirical evidence exploring the relation between network maturation and structural changes over longer periods of time is thus needed. In other words, what does a shift from coordination to cooperation as the main collective action problem in a maturing network mean in terms of measurable structural features of the underlying social network?

We explore how changes in the nature of risk associated with network maturation are reflected in the network structure by focusing on two structural mechanisms. Closure and centralization are two mechanisms that are able to characterize a large amount of the quality of structural changes in a network. Observing changes in centralization and closure offers a parsimonious way to test hypotheses about the expected development of a network based on the general risk hypothesis.

Network centralization as an outcome of centralization processes is a network-level measure which describes the degree in which a graph is centered around a certain focal point (Scott 2013, 90). Put another way, it indicates how hierarchically a network is structured (Carlsson and Sandström 2008, 41). In our treatment of centralization, we focus on degree centralization, which measures the inequality of tie distribution in the network. High levels of centralization play an important role in low-risk dilemmas generally associated with coordination problems. At this point in time, star-like structures are expected to emerge, with central actors providing efficient coordination. In a more high-risk setting, centralization is expected to decrease because actors are becoming weary of the possibility that other actors with a central coordination role are getting able to exploit their brokerage role in the network. Thus, by choosing to form ties that lead to an overall decrease in centralization actors react to the risk that comes with a dependence on non-redundant connections implied by centralization (Berardo and Scholz 2010).

However, these assumptions could not be upheld in a recent test of the risk hypothesis in a high- 
risk socio-ecological system (Berardo 2014). One interpretation of this surprising finding is that even in high-risk situations, the local ecology of games is characterized by a mixture of cooperation and cooperation dilemmas. As our analysis involves a maturing network over a longer time period we should be able to shed more light on these contradictory initial findings. A longitudinal analysis of the network allows doing this by focusing on the processes of change in a network instead of relying on absolute measurements at a certain point in time.

A focus on change over time is important because even if a network in a high-risk setting shows low centralization, this does not necessarily mean that no processes of centralization are at play at this particular stage of the evolution of the network. Centralization might have been even lower before. As a consequence, even though a more mature stage of the network might still be characterized by some amount of mixture between coordination and cooperation dilemmas, we still expect an overall decreasing influence of centralization as the network matures in the longer term:

H1. Long-term network development in maturing networks for natural resource governance is driven by decreasing centralization.

Network closure has a long history of being recognized as a source of bonding social capital. This rests on the assumption that cohesive networks where people are connected through redundant and strong connections create trust and security. As everyone can keep an eye on everyone else, threats of sanctions become credible (Burt 2000). A very important measure of closure is transitivity. Transitivity as an outcome of the process of transitive closure measures the extent to which members of a dyad share partners and how those partners are connected to each other (Robins, Bates, and Pattison 2011, 1297). Transitivity has been an important concept following the works of theorists such as Granovetter (1973). The continuing occupation with the concept stems from the fact that it is the most essential feature that differentiates random networks from observed data (Snijders et al. 2006, 100). As a network matures, we expect actors to rely more on bonding social 
capital in response to the increase in cooperation problems, which will lead to an increase in the process of transitive closure.

H2. Long-term network development in maturing networks for natural resource management is driven by increasing transitive closure.

Thus, overall, we expect network development to be driven by increasing transitive closure and decreasing network centralization.

\section{Controlling for non-structural effects}

Beyond structural effects based on network self-organization, the development of the network is likely to be shaped by two main groups of effects based on individual actor behavior. Consequently, actor attributes might emerge as additional drivers of network development through activity and homophily effects:

Activity can generally be measured by the number of ties an actor forms. Some types of actors might be more active than others, leading to their over-representation in the total of connections. It is often assumed that especially state and federal agencies tend to show high activity in governance networks because of their specific capacities and resources, ranging from access to expertise to political authority (Lubell, Robins, and Wang 2014). Empirically, this effect has recently been found in short-term governance networks for conservation in Finland (Borg, Toikka, and Primmer 2015). Thus, one possible driver of network development might be a high activity of higherlevel government actors. This might especially be the case in nascent networks as higher-level government agencies are ideally suited to take up the central roles in the hypothesized star-like structures. However, as a networks matures, network structure might be increasingly driven by activities of private sector and lower-level actors. This would reflect the overall design principles of multi-level network governance arrangements. 
Perhaps the most well-discussed observation in research on how attributes of actors lead to mechanisms that influence network structure is homophily. Homophily is the principle that a contact between similar actors occurs at a higher rate than among dissimilar actors (Mcpherson, SmithLovin, and Cook 2001, 416). This means that similar actors will have a higher tendency to form contacts. They also might become more similar over time as well in a self-reinforcing dynamic (Newman and Dale 2006). One reason for this is that common norms and values develop in deepening social relationships (Bodin and Crona 2009, 372). Assortative mixing, the process leading to homophily, might assume a prominent role as the network matures, because actors start building more bonding social capital, leading them to seek ties to similar alters. 


\section{Empirical test of dynamic hypotheses in a regional nature park}

In order to test the hypotheses we have derived from our theoretical foundations, a test case must fulfill three key conditions. First, the network in question must have existed for a sufficiently long time after its creation, preferably a number of years, to go through a substantial evolution. This enables us to compare changes over time between nascent and mature stages of the network, as envisioned. Secondly, the network should represent a governance network established to manage natural resources. Thirdly, the governance network should be delimited by a relatively stable set of actors that allows for an empirical investigation of their network relations over time.

The governance network surrounding the regional nature park of Thal fulfills these conditions. We have been able to observe its development over more than six years, changing from a nascent network working when the park project was set up to a mature network that focuses today on the enabling and maintaining the cooperation of its various actors in the day-to-day operations of the park. Further, even though network has changed in its composition over time, the orientation towards the establishment and operation of the regional park project under a common park label allows us to understand it as one governance network that has developed over time.

Regional nature parks (RNPs) are an integral part of the national Swiss park strategy, which, in turn, is an essential part of Swiss regional development and landscape policy. The legal foundations for the establishment of parks can be found in a revision of the Federal Act on the Protection of Nature and Cultural Heritage, revised in 2007. It gives the federation the possibility to support the establishment, operation and quality control of regional park projects with federal funding. The federation assigns successful park projects with a label that is awarded based on a set of criteria and requires that a park project is initiated by local stakeholders in the region. As such, the park policy attempts to position park regions as nationally and internationally recognized areas of maintained and enhanced landscape and nature quality. At the same time, parks also should enhance sustainable regional development with broad participation of the various actors present in 
a particular region (Federal Office for the Environment 2010).

Problems that rural regions in Switzerland face include the diminishing importance of traditional economic sectors such as forestry or agriculture, alongside the gravitational pull of urban areas for the increasingly important service sector. This may lead to the marginalization and depopulation of rural regions. It leaves Swiss rural regions in a dilemma: tourism is oftentimes the only area of potential growth left in many areas, but intensive land use - partly due to tourism can degrade the very landscape which is the source of a region's touristic potential (Hirschi 2010). RNPs are an innovative way to manage landscape as a resource on a more appropriate landscape scale, transcending traditional administrative boundaries (Gerber and Knoepfel 2008).

Actors in the Thal region recognized early on the suitability of the region for a RNP. After efforts had been put underway, the region was the first RNP to be awarded the federal park label in 2009. As the oldest of its kind, the RNP of Thal thus makes for an excellent test case for the study of a social network in natural resource governance from a longitudinal perspective.

\section{Research design}

To trace the development of the governance network surrounding the RNP of Thal, network data from two observations are analyzed. The first observation (t1) took place during initiation of the park project in 2008. A survey of the complete network was carried out right after the region had officially submitted its park project to the federal government (Hirschi 2010). We interpret this stage of network development as the nascent phase, where for most actors no substantial risk was involved in the venture as concrete effects of the projects were not yet evident. Therefore, the main types of problems present in the network were coordination dilemmas as the design proposed by the federation demanded the viewpoints of all relevant actors needed to be integrated.

The survey was carried out again for a second observation (t2) in the first half of 2014. By then, the park project had been in existence for more than six years. In the meantime, the RNP had 
entered a more mature stage, dominated by readjustments and reflections about goal attainment. The process of applying to the federal government for the extension of the park project, due for every park project after ten years of existence, had also already started to become a topic. We interpret this mature stage as one where the risk for actors in the network is substantially higher than in the nascent phase. Involvement in the project now had tangible consequences as more actors were present that were also more invested (especially financially) and expectations for the park project to show concrete results were higher. This implies an overall increase in cooperation problems.

While planning the empirical study, we put particular emphasis on maximum comparability between the two network observations. For one, this meant that the network boundaries were defined in a way that the same network was captured as well as possible at the two points in time. Setting a network boundary is always a theoretically informed decision. The social construction of relations means that social networks do not have boundaries which can be obviously identified (Scott 2013, 44). The use of a bottom-up approach to data collection has in the past been described as problematic because of its high reliance on the perceptions of participants. This puts it in danger of neglecting indirect or direct factors that affect the behavior of actors but are not recognized by them (Sabatier 1986). We have tried to overcome these difficulties by combining several methodological approaches:

To establish the network boundary for $\mathrm{t} 1$, a positional approach was chosen that identified actors based on formally defined positions and group memberships (Scott 2013, 45) by investigating written documentation and conducting exploratory interviews with key actors in the region. For $\mathrm{t} 2$, this approach was repeated, but additionally involved a combination of further methods to identify relevant actors: first, and as a basis for the further procedure, the existing list of actors was reviewed and checked to ascertain whether the actors still existed; secondly, additional possible actors involved in the management and development of the park were identified based on written documentations; thirdly, a reputational approach (Scott 2013, 46) was used to refine the final net- 
work boundary. The augmented list of actors was reviewed by both the project leader of the park and a former scientific advisor to the park project.

This procedure resulted in a network consisting of 36 actors in 2008 (t1). By 2014, the network had grown to 58 actors. Between 2008 and 2014, seven actors left the network, 31 actors remained involved and 27 new actors entered the network. The new actors display certain regularities. First, a very important new actor is the park project management team itself, which took up its work after 2008. Second, a crucial group of new actors consists of local restaurants and hotels as well as producers of regional products who have entered label partnerships with the nature park management. Beyond this, a number of interest and advocacy groups at all levels have gotten involved over the years.

In 2008 (t1), a standardized questionnaire was sent to all 36 actors by regular mail (response rate: 89\%). In 2014 (t2), a standardized online survey was carried out for all 58 actors (response rate: $81 \%$ ). In both surveys, actors were asked to nominate actors from a list of all other actors in the network with whom they closely collaborated during the establishment (t1) and management of the park (t2), respectively. This information was then used to create a undirected network of collaboration ties. A tie was established based on a maximum criterion whenever one of the respondents indicated collaboration. The approach chosen to establish the network boundary seems to have captured the network in question well. Respondents were explicitly asked to name relevant organizations which were missing from the list in their opinion to further validate the chosen network boundary. Only nine respondents reported further actors which had not been included in the survey beforehand. Among those, only the major supermarket chain in the region and a regional product distributor were named as new actors more than once.

All the network actors were then coded for the attributes jurisdictional level ${ }^{1}$, actor type ${ }^{2}$ and dimension ${ }^{3}$.

\footnotetext{
${ }^{1}$ Level differentiates between local, regional, state and national actors.

${ }^{2}$ Type includes the categories public, private and civil society.

${ }^{3}$ Dimension refers to dimensions of sustainable regional development based on the stated objectives of the park project. An organization was only assigned a certain dimension if it could be clearly and exclusively placed within one
} 
In both observations, actors do not represent individuals but corporate actors (Coleman 1974), representing organizations such as public agencies, firms and civil society organizations. Governance networks are usually viewed from this perspective. However, there are a number of potential issues in translating concepts that originated from research on interpersonal networks to the the study of corporate actors. For example, it is not always clear what concepts such as homophily and trust mean when applied to organizations (Newig, Günther, and Pahl-Wostl 2010). Recognizing this as an important caveat, we still believe it does not substantially influence our study. The ecology of games approach, as the theoretical foundation of our study, is based on a game-theoretical approach that assumes that a large part of the behavior of actors can be explained by rational behavior. Assuming that corporate actors rationally pursue their interests just as individuals do, this does mean that there is not a great difference between interpersonal and organizational network dynamics in this regard. Processes of homophily between organizations can also be plausible. We measure homophily on the above named actor attributes of actor types, jurisdictional level and thematic dimension. On all of these categories it seems reasonable that corporate actors would be more inclined to form ties with similar actors, i.e. actors with similar organizational characteristics, for example based on shared organizational goals (nature protection versus regional products) or inter-organizational structures (public agencies versus firms) that make collaboration easier.

\section{Methods}

To test our hypotheses we turned to statistical modeling which allows for inferences about the presence or absence of structural network characteristics (Robins, Bates, and Pattison 2011, 1307).

Exponential random graph models (ERGMs) can model network structure and social selection effects. ERGMs make it possible to make statistical inferences about the configuration of

dimension. The regional economic dimension was split up in regional products and restaurants/ hotels to allow for a more fine-grained treatment of these actors representing different economic sectors. Dimensions, thus, were tourism, nature protection, regional products/ other economic interests and restaurants/ hotels. 
a network, allowing for statements on whether a given configuration occurs more than could be expected by chance (Robins, Lewis, and Wang 2012, 386). Fundamentally, ERGMs can be understood as complex auto-logistic regressions that predict the presence or absence of a tie in a network. However, the main difference from logistic regression models is that network tie variables are not assumed to be independent. The presence or absence of a tie is, thus, predicted from the patterns of other ties. This makes network ties predictors and response variables at the same time (Robins, Bates, and Pattison 2011, 1307). This way, ERGMs allow for the inclusion of structural, dyadic-dependent effects such as tendencies towards closure or centralization in the development of a network over time. These effects allow to capture these two main dependent variables of this paper.

At the same time ERGMs make it possible to control for dyadic-independent effects such as homophily (for example a greater than expected tendency of two state actors to collaborate). As mentioned above, this distinction between dyadic-dependent and dyadic-independent effects is crucial and necessitates the use of a procedure such as ERGM. As long as the two types of effects can not be untangled, it is very hard to be reasonably sure that observed structural effects are truly structural. For example, an observed increase in transitivity (indicating bonding social capital) in a network does not need to be a result of network-wide closure. It could also to a large part be driven by the presence of a small group of actors that are simply very active in creating ties.

All calculations were carried out in the R package statnet (Handcock et al. 2003). To make a longitudinal statistical analysis of changes in the network possible, a separable temporal exponential random graph model (STERGM) was estimated. STERGMs mark a recent refinement in the modeling of dynamic networks. Up to now, most models have been able to interpret information about the prevalence of certain network properties at a given point in time. This could for example concern the level of centralization in a network. However, with time series data consisting of a number of snapshot of a given network it is possible to go beyond observing changes in the prevalence of a given property. Time series contains information that allows to analyze the dynamic 
processes that produce the prevalence of a given property.

In a technical development following these considerations, STERGMs thus differentiate between incidence and duration in fitting a model to longitudinal data. Considering the example of centralization, information about incidence would capture the rate at which new ties formed that contributed to centralization. Duration on the other hand would look at how long these ties lasted. Both of these processes together in the end influence the prevalence of centralization at a given point in time (Krivitsky and Handcock 2014). Understanding prevalence as a composite of incidence and duration offers valuable insights into the development of a network.

In the context of this paper, the STERGM model provides answers about the processes that govern the creation and breaking up of ties and thus allows for a profound test of the formulated hypotheses.

\section{Results}

In line with the current state of the art for the identification of network self-organization based on tie dependence variables, we fit a basic social circuit dependence model. Thus, we specify model terms based on the geometrically weighted statnet variants of configurations (as developed by Snijders et al. 2006) and suggested by Robins, Lewis, and Wang (2012, 387). The models contain four structural parameters for network self-organization:

- An edge parameter represents the baseline propensity for ties to form in a network. This parameter is usually negative for most observed networks (Harris 2014).

- An activity spread (centralization) parameter, based on k-star (degree) effects using the geometrically weighted degree (GWDegree) term implemented in statnet. GWDegree is an anti-preferential attachment term (Hunter 2007). A positive parameter thus suggests a more even share of degree among actors. For a negative parameter value, ties from low to high 
degree actors are more likely. However, due to the geometrical weighting, the gains in this effect decrease rapidly for increasing degree values (Snijders et al. 2006, 114).

- A multiple triangulation (closure) parameter, which is based on k-triangles or alternating triangles. A positive estimate is an indicator of transitive closure effects (Robins, Lewis, and Wang 2012, 388). In statnet, this parameter is implemented in the geometrically weighted edgewise shared partner (GWESP) term.

- For the multiple triangulation parameter to adequately capture transitive closure, it is necessary to include a measure of multiple connectivity. An inclusion of such a measure makes it possible to separate transitive closure from an accumulation of many two-paths happening by chance (Snijders et al. 2006, 122). In statnet, this is implemented via the geometrically weighted dyadwise shared partners (GWDSP) term (Hunter 2007). The GWDSP term can be understood as a prerequisite in order to able to properly interpret whether the multiple triangulation parameter faithfully captures closure.

Two groups of parameters are used to control for effects based on actor attributes. We use homophily parameters to control for assortative mixing effects influencing the propensity of ties to form among actors which share the same type, level or dimension. Activity parameters reflect the consideration that groups of actors based on actor attributes might display a higher or lower than average baseline propensity to form ties.

In a single STERGM modeling procedure, two models are always specified: The formation model makes it possible to assess what governs the creation of ties between the two observations. The dissolution model does the same for the likelihood of ties to be broken off.

The $\alpha$ parameter for the geometrically weighted structural parameters were independently fixed for both the formation and dissolution model ${ }^{4}$. Model building consisted of starting with a simple

\footnotetext{
${ }^{4}$ For the formation model $\alpha$ values were fixed at .1 (GWDegree), 1.5 (GWESP) and .6 (GWDSP). For the dissolution model $\alpha$ values were fixed at .2 (GWDegree), 1.8 (GWESP) and .3 (GWDSP).
} 
model for both models containing only structural effects and adding attribute-based effects in order to increase model fit. Afterwards, non-significant effects were gradually removed from the models in order to arrive at the final model specifications. The final models converged well during estimation. Figure 1 shows a further examination of goodness of fit measures based on a simulation of 1000 randomly generated networks. The formation as well as the dissolution model reproduce distance and triad census very well. The formation model is also remarkably well able to capture the degree distribution, given its erratic behavior. Under 'model statistics' the figure also gives further indication of good convergence. The observed statistics for all model terms are generally near the median of the of the plotted quantiles of the simulated sample. This indicates that the simulated networks capture the observed network well on most of the model terms.

Table 1 shows the parameter estimates for the final models. For tie formation, the parameters show significant estimates for all structural effects except for centralization. Tie formation based on structural effects is mainly driven by tendencies towards closure. This is implied by the positive multiple triangulation and connectivity estimates.

For the attribute-based variables the results show a highly significant regional activity effect. The effect indicates that ties are more likely to form over time if they involve a regional-level actor. Also statistically significant, but weaker, is a homophily effect for type and level, denoting that ties are slightly more likely to form between actors of the same type, such as between government actors or between private sector actors, and between actors at the same jurisdictional level. Additionally, the model captures a higher activity of private sector, regional and state level actors in forming ties.

As for the dissolution model, it is important to note that the parameters are measures of persistence. Positive parameters, hence, imply longer tie duration (Krivitsky and Handcock 2014, 41). The only, but highly significant estimate for structural effects besides the edge parameter occurs for multiple triangulation. Here, the positive estimate implies that ties are much more likely to persist if they contribute to closure. Besides this effect, there are only two other attribute based effects that 
are important in explaining tie dissolution. First, there is a strong tendency for ties including local and state level actors to last longer, indicated by the positive and highly significant state activity effect. Second, there is some indication that this also holds for ties involving local level actors, although the effect is weaker and less significant. However, the results of the dissolution model should not be overinterpreted, as the variance in tie dissolution is much lower than in tie formation due to the generally growing network over time. This low variance in tie dissolution also explains the absence of homophily terms in the dissolution model because only a very small number of homophilous ties were lost and could not be modeled.

\section{Discussion}

The general predictions for the development of centralization and bonding social capital reflected in hypotheses $\mathrm{H} 1$ and $\mathrm{H} 2$ are partially supported by the statistical analysis, with some surprising results.

Hypothesis $\mathrm{H} 1$ can not be supported by the results of the modeling procedure. There is no recognizable trend towards decreasing centralization in the development of the network. But at the same time the opposite can also not be found. It seems that centralization, if it occurs, does not play a significant part in shaping the development of the network over time and is overshadowed by other dynamics.

However, a large and statistically robust effect in connection with our hypotheses can be found for the influence of closure on tie formation. This supports the hypothesis H2. There is a clear indication that ties are more likely to form if they contribute to closure, based on the formation model. Also, ties are more likely to persist if they contribute to closure.

Beyond these structural effects, the results show some interesting further trends in dyadicindependent effects. Actor attributes turn out to play an important role in shaping network development. 
A large attribute-based effect for the formation of ties can be found for the activity of regional level actors in forming new ties. As the governance network matures, there is evidence that regional level actors are significantly more active in forming new ties. Regional level actors are thus much more active in expanding the number of organizations they collaborate with during network development. This fits in with other studies that have also found regional actors to play a central role in comparable empirical settings due to their role as potential gatekeepers between local and higher level actors (Ingold 2014). Private sector actors play a similar role. During network maturation they are getting much more involved in regional governance which could be explained by the increasing risk of non-participation as activities in the network shift from planning to actual implementation. In the case of the Thal region, this is also very much due to the increasing involvement of local restaurants entering labeling partnerships with the park project, which was controlled for in the model (not shown).

After accounting for structural effects and activity based on actor attributes, it becomes clear that a considerable amount of tie formation remains that is driven by homophily effects. There are significant effects of a higher likelihood of ties between similar actors to emerge on all included dimensions of homophily. This speaks for the propensity of actors to first choose similar others to collaborate with over the course of network development. The finding could be explained by a perceived lower risk associated with joining like-minded and close actors in collaborative undertakings.

To summarize, the regional nature park of Thal has provided a rare chance to gather empirical data for the development of a governance network for natural resource management. The empirically observed governance network generally shows some of the expected development patterns of a maturing network but holds some surprises in others. On the one hand, it has been characterized by a continuing drive toward transitive closure, building crucial bonding social capital for cooperation in resource management. On the other hand, there is no tendency toward less centralization recognizable over time. The network is not becoming less hierarchical as early discussions of the 
implications of the risk hypothesis in a longitudinal setting have suggested (Berardo and Scholz 2010). Instead our findings add additional evidence to the findings of an earlier study by (Berardo 2014) in a longitudinal setting.

We see two potential factors that could be responsible for this persistence of centralization. The first is an ongoing presence of coordination problems requiring efficient centralized information gathering besides an increase in the amount of cooperation problems. In the case of the park project, this could be due to the more coordinative endeavor of readjusting the project to apply for extended federal funding. The second factor is a potential disregard for risks associated with a dependence on highly centralized overall structures by individual actors. This could on the one hand be due to a high level of trust in the group of highly central actors. On the other hand it could be based on a perceived mitigation of risk by increased and more localized closure at the level of individual actors. This would outweigh the risks they associate with a dependence on few central actors, especially because centralized structures have advantages in other areas, such as efficient information gathering.

There are some limitations of the analysis that should be mentioned:

First, of course, we cannot generalize our findings based on the analysis of one network alone. However, the design of our study is potentially comparable to others and, thus, allows for some cross-sectional comparisons.

Secondly, there might have been an unidentified change in the content of the ties between the two observations. The content of ties is an aspect of networks which can evolve besides their structure (Bodin and Crona 2009). The study design aimed to maximize comparability between two observation periods on this count by various means. Still, the meaning of close collaboration in the specific context might have undergone a transformation with unknown implications for the overall structure.

Thirdly, difficult ties are not covered by the analysis. This again concerns the content of network ties. The analysis did not specifically explore whether there were relationships characterized 
by very difficult close collaboration. An integration of difficult or negative ties however can illuminate interesting questions about fragmentation and difficulty in a network (Robins, Bates, and Pattison 2011).

Fourthly, the analysis undertaken has mostly stayed on a "birds-eye" level of overall structural characteristics. It could also be complemented by exploring more qualitative attributes of network structure. This could involve a more detailed profiling of actor attributes of central organizations, listing their preferred strategies or characteristics of board members. Such attributes have been shown in the past to play a role in the outcomes of network governance (Bodin and Crona 2009, 370). 


\section{Conclusion: Effective governance in an evolving network}

In this paper, we have shed light on a topic that has received scant empirical and only sporadic theoretical treatment in the literature on natural resource governance. Building on recent developments both methodological and theoretical, we have been able to develop and test hypotheses about trends regarding centralization and bonding social capital in maturing social networks involved in natural resource governance. The EG framework has proven to be very useful for the undertaking of this study, showing great potential to harness its capacities for generating and testing hypotheses in further studies.

However, the main motivation behind the continuing interest in networks in the field of natural resource governance is to establish features which are associated with effective governance. The results of this study have added an important element to these considerations by explicitly studying the role of network dynamics. For the two crucial structural characteristics of bonding social capital and centralization, some dynamics during network maturation are clearly recognizable.

What are the implication of these dynamics for ensuring continuous effectiveness of natural resource governance? We see two main points that should be taken into account.

First and foremost, it is crucial to acknowledge the implications of the fact that the challenges of natural resource governance as well as social networks are both dynamic. This means that the way in which the structure of a governance network contributes to effectiveness has to be analyzed related to its maturity. We have shown that that the tools to do this are available and should be made use of more often.

Second, we have observed shifts in network structure that accompany the maturation of the network. Judging from one study alone, we can not be sure if these shifts have also been associated with upholding governance effectiveness over time. It has been beyond the confines of this study to thoroughly assess the network surrounding the regional nature park of Thal on its effectiveness. However, actors in the region were successful in setting up the park in the first place and secur- 
ing initial federal funding. Adding on to this, actors in the network have successfully switched from setting up the park to managing it in the following years, putting their plans and visions into practice. The project has also been meeting the expectations of the federal government and has thus recently been guaranteed funding for the continuation of the project up to 2019. Actors in the region have also been satisfied enough with the project to agree to its continuation in the coming years. This is something that not all Swiss regional nature park projects have managed to do. On these counts, the network has already been effective. It does not go to far then to say that the generation of bonding social capital and the upkeep of some degree of centralized coordinative roles emerge as very promising structural processes for continuing effectiveness during the maturation of a network for natural resource governance.

These findings, generated from an in-depth analysis of a single case obviously need to be backed up by further studies involving more cases. Importantly, effectiveness or performance would also need to be assessed on a more profound level. We have connected network dynamics and effectiveness to some degree in this study, but future studies would need to look at the dynamic relationship between function, structure and performance simultaneously and in more detail.

Beyond trends in bonding social capital and centralization, this study has also taken into account the influence of specific groups of actors on network development. This made it possible to generate potential hypotheses for further exploration. To conclude, we want to highlight two groups of actors that shape network development we consider especially interesting:

The true extent of local-level involvement within a given policy design should be further scrutinized. Contrary to what is generally expected due to their specific capacities, higher-level government agencies did not emerge as a central driving force in network development in our study. Instead, private sector and regional level actors were more active in forming the network. One possible explanation of this phenomenon is that private sector actors might hesitate to devote their costly time to getting involved in a network during its creation. However, once the governance network has become operable and starts having concrete effects on the ground, they start getting 
involved. Another explanation is the strong bottom-up character of the park project studied in this paper. Put another way, the question remains how much of local, non-government actor involvement is due to policy program design and how much of it reflects a general pattern of change during network maturation? This is a question that is also relevant to policy-makers trying to achieve local stakeholder participation in natural resource governance. It shows that such participation might potentially be easier to achieve at later stages of a project.

The example of state-level actors shows the insights that can be gained by taking a close look at the roles different actor groups play within a network. While state-level actors were found to be active in forming connections in our study, they were, however, even more influential in supplying lasting, persistent ties within the network. This points toward a possible refinement of their role during the evolution of a network. As a network matures, they take on a new and important role in providing stable, reliable connections within the network. As demonstrated in this paper, separable temporal models have a lot to offer in making such differentiated actor roles visible.

The overarching story of this paper has been one of adding depth to the role of networks in natural resource governance by integrating a dynamic perspective. But, of course, network structure is one among many factors when it comes to explaining the performance of a governance system. Macro-level institutions, individual decision-making and broader societal and economical developments interact with each other and impact governance outcomes. And they do it in a complex setting of social-ecological interdependences and cultural framing.

\section{References}

Anderies, John M., Marco A. Janssen, and Elinor Ostrom. 2004. "A Framework to Analyze the Robustness of Social-ecological Systems from an Institutional Perspective.” Ecology and Society 9 (1): 18. 
Armitage, Derek, Melissa Marschke, and Ryan Plummer. 2008. “Adaptive co-management and the paradox of learning." Global Environmental Change 18 (1): 86-98.

Berardo, Ramiro. 2014. “The Evolution of Self-Organizing Communication Networks in HighRisk Social-ecological Systems." International Journal of the Commons 8 (1): 236-258.

Berardo, Ramiro and John T. Scholz. 2010. "Self-Organizing Policy Networks: Risk, Partner Selection, and Cooperation in Estuaries.” American Journal of Political Science 54 (3): 632-649.

Bodin, Örjan and Beatrice I. Crona. 2009. "The Role of Social Networks in Natural Resource Governance: What Relational Patterns make a Difference?." Global Environmental Change 19 (3): 366-374.

Borg, Riikka, Arho Toikka, and Eeva Primmer. 2015. "Social capital and governance: a social network analysis of forest biodiversity collaboration in Central Finland." Forest Policy and Economics 50, 90-97.

Burt, Ronald S. 2000. “The Network Structure of Social Capital.” Research in Organizational Behavior 22 (1): 345-423.

Carlsson, Lars and Annica Sandström. 2008. "Network Governance of the Commons." International Journal of the Commons 2 (1): 33-54.

Coleman, James S. 1974. Power and the structure of society. New York: Norton.

Ernstson, Henrik, Sverker Sörlin, and Thomas Elmqvist. 2008. "Social movements and ecosystem services-The role of social network structure in protecting and managing urban green areas in Stockholm.” Ecology and Society 13 (2): 39.

Federal Office for the Environment. 2010. Pärke von nationaler Bedeutung, Richtlinie für Planung, Errichtung und Betrieb von Pärken. Bern: FOEN.

Folke, Carl, Thomas Hahn, Per Olsson, and Jon Norberg. 2005. “Adaptive Governance of Socialecological Systems." Annual Review of Environment and Resources 30 (1): 441-473.

Gerber, Jean-David and Peter Knoepfel. 2008. “Towards Integrated Governance of Landscape Development." Mountain Research and Development 28 (2): 110-115. 
Gerring, John. 2010. "Causal Mechanisms: Yes, But...." Comparative Political Studies 43 (11): $1499-1526$.

Granovetter, Mark. 2005. "Impact of Social Structure on Economic Outcomes." The Journal of Economic Perspectives 19 (1): 33-50.

Granovetter, Mark S. 1973. "The Strength of Weak Ties.” American Journal of Sociology 78 (6): $1360-1380$.

Handcock, Mark S., David R. Hunter, Carter T. Butts, Steven M. Goodreau, and Martina Morris. 2003. statnet: Software tools for the Statistical Modeling of Network Data. Seattle, WA.

Harris, Jenine. 2014. An Introduction Exponential Random Graph Modeling. London: Sage Publications.

Hirschi, Christian. 2010. "Strengthening Regional Cohesion : Collaborative Networks and Sustainable Development in Swiss Rural Areas." Ecology and Society 15 (4): 16.

Hunter, David R. 2007. “Curved Exponential Family Models for Social Networks.” Social networks 29 (2): 216-230.

Ingold, Karin. 2014. "How involved are they really? A comparative network analysis of the institutional drivers of local actor inclusion." Land Use Policy 39, 376-387.

Janssen, Marco A., Örjan Bodin, John M Anderies, Thomas Elmqvist, Henrik Ernstson, Ryan R. J. Mcallister, Per Olsson, and Paul Ryan. 2006. "Toward a Network Perspective of the Study of Resilience in Social-ecological Systems." Ecology and Society 11 (1): 15.

Kenis, Patrick and Keith G. Provan. 2009. "Towards an Exogenous Theory of Public Network Performance." Public Administration 87 (3): 440-456.

Krivitsky, Pavel and Mark Handcock. 2014. “A Separable Model for Dynamic Networks.” Journal of the Royal Statistical Society: Series B (Statistical Methodology) 76 (1): 29-46.

Lubell, Mark. 2013. "Governing Institutional Complexity: The Ecology of Games Framework." Policy Studies Journal 41 (3): 537-559. 
Lubell, Mark. 2015. “Collaborative Partnerships in Complex Institutional Systems.” Current Opinion in Environmental Sustainability 12 (1): 41-47.

Lubell, Mark, Garry Robins, and Peng Wang. 2014. "Network Structure and Institutional Complexity in an Ecology of Water Management Games.” Ecology and Society 19 (4): 23.

Lubell, Mark, John Scholz, Ramiro Berardo, and Garry Robins. 2012. “Testing Policy Theory with Statistical Models.” Policy Studies Journal 40 (3): 351-374.

Mcpherson, Miller, Lynn Smith-Lovin, and James M. Cook. 2001. “Birds of a Feather: Homophily in Social Networks.” Annual Review of Sociology 27 (1): 415-444.

Newig, Jens, Dirk Günther, and Claudia Pahl-Wostl. 2010. "Synapses in the Network: Learning in Governance Networks in the Context of Environmental Management." Ecology and Society 15 (4): 24.

Newman, Lenore and Ann Dale. 2006. "Homophily and Agency: Creating Effective Sustainable Development Networks." Environment, Development and Sustainability 9 (1): 79-90.

Ostrom, Elinor. 2011. "Background on the Institutional Analysis and Development Framework." Policy Studies Journal 39 (1): 7-27.

Robins, Garry, Lorraine Bates, and Philippa E. Pattison. 2011. "Network Governance and Environmental Management: Conflict and Cooperation." Public Administration 89 (4): 1293-1313.

Robins, Garry, Jenny M. Lewis, and Peng Wang. 2012. "Statistical Network Analysis for Analyzing Policy Networks." Policy Studies Journal 40 (3): 375-401.

Sabatier, Paul A. 1986. “Top-Down and Bottom-Up Approaches to Implementation Research: a Critical Analysis and Suggested Synthesis.” Journal of Public Policy 6 (1), 21-48.

Scott, John. 2013. Social Network Analysis. London ; Thousand Oaks, Calif.; New Delhi: Sage Publications.

Snijders, Tom A., Philippa E. Pattison, Garry L. Robins, and Mark S. Handcock. 2006. "New Specifications for Exponential Random Graph Models.” Sociological Methodology 36 (1): 99153. 
Snijders, Tom A., Gerhard G. van de Bunt, and Christian E. G. Steglich. 2010. "Introduction to Stochastic Actor-Based Models for Network Dynamics.” Social Networks 32 (1): 44-60.

Underdal, Arild. 2010. "Complexity and challenges of long-term environmental governance." Global Environmental Change 20 (3): 386-393.

Walker, Brian, C. S. Holling, Stephen R. Carpenter, and Ann Kinzig. 2004. "Resilience, Adaptability and Transformability in Social-ecological Systems." Ecology and Society 9 (2): 5. 


\begin{tabular}{|c|c|c|c|c|}
\hline \multirow[t]{2}{*}{ Parameter } & \multicolumn{2}{|c|}{ Formation } & \multicolumn{2}{|c|}{ Dissolution } \\
\hline & Estimate & Standard Error & Estimate & Standard Error \\
\hline \multicolumn{5}{|l|}{ Structural } \\
\hline Edges & $-7.09^{* * *}$ & 0.36 & $-3.45^{* * *}$ & 0.92 \\
\hline $\begin{array}{l}\text { Activity spread } \\
\text { (centralization) }\end{array}$ & 0.21 & 1.34 & -0.98 & 1.02 \\
\hline $\begin{array}{l}\text { Multiple triangulation } \\
\text { (closure) }\end{array}$ & $0.81^{* * *}$ & 0.07 & $0.45^{* * *}$ & 0.12 \\
\hline Multiple connectivity & $0.10^{* * *}$ & 0.01 & $0.20^{* *}$ & 0.06 \\
\hline \multicolumn{5}{|l|}{ Homophily } \\
\hline Same type & $0.54^{* * *}$ & 0.16 & & \\
\hline Same level & $0.46^{* *}$ & 0.15 & & \\
\hline Same dimension & $0.38^{*}$ & 0.17 & & \\
\hline \multicolumn{5}{|l|}{ Activity } \\
\hline Private sector activity & $0.77^{* * *}$ & 0.12 & -0.66 & $(0.82)$ \\
\hline Local activity & & & $0.80^{*}$ & 0.34 \\
\hline Regional activity & $0.57^{* * *}$ & 0.11 & & \\
\hline State activity & $0.42^{* * *}$ & 0.13 & $1.17^{* *}$ & 0.35 \\
\hline
\end{tabular}

${ }^{* * *} p<0.001,{ }^{* *} p<0.01,{ }^{*} p<0.05$

Table 1: Parameter estimates for separable temporal random graph model of close collaboration network 